\title{
Las demandas informativas de los inmigrantes latinoamericanos en la Comunidad de Madrid
}

\section{Jorge Cardoso Castro}

Profesor de Estilos y Géneros de Opinión.

Universidad CEU San Pablo

\section{José Alberto de Francisco Rodríguez}

Profesor de Comunicación e Información Audiovisual.

Universidad CEU San Pablo

\section{Viviana Fernández Marcial}

Profesora de Documentación Informativa.

Universidad CEU San Pablo

Resumen:

La presente investigación, financiada por la Universidad San Pablo-CEU, tiene como objeto de estudio fundamental el análisis de las demandas informativas de los inmigrantes latinoamericanos en la Comunidad Autónoma de Madrid. Sus objetivos específicos han sido averiguar cuáles son las aspiraciones o metas de los sujetos, sus necesidades y deseos, sus problemas y preocupaciones, así como las aficiones, tiempos y espacios de ocio. De los resultados de todo ello se han podido identificar los objetos de referencia o demandas informativas de este colectivo, en función de sus temas de interés, que han sido los siguientes: la legislación española de extranjería, el trabajo, el paro, la vivienda, el dinero, los estudios, la integración social, la música y baile, y el fútbol. A partir de estos resultados se tiene previsto llevar a cabo una segunda investigación sobre las fuentes de información y usos de la comunicación por parte de este mismo colectivo de inmigrantes.

Palabras clave:

Demandas informativas - inmigrante - latinoamericanos - Comunidad de Madrid - aspiraciones - necesidades - preocupaciones - ocio 


\section{Abstract:}

The research, supported by San Pablo-CEU University, is focused on what kind of news the Latin-American immigrants want to now in Madrid. Finding out their main goals, their needs and desires, their problems and also the hobbies and free times, are the objectives. It is of the results of all it they have been possible to identify the objects of reference or informative demands of this group, based on his subjects of interest, that they have been the following ones: the Spanish lasws of foreigner, the work, unemployment, the house, the money, the studies, social integration, music and dance, and soccer. From these result it is had predicted to carry out one second investigation on the sources of intelligence and uses of the communication on the part of this same group of migrants.

Key words:

Informative demands - immigrants - Latin Americans - Madrid (autonomous community) - aspirations needs - worries - free time

\section{Introducción}

El fenómeno de la inmigración en Europa se ha convertido en los últimos años en una de las cuestiones prioritarias de los gobiernos de los países miembros de la UE. En el caso de España, este fenómeno ha adquirido dimensiones insospechadas. En las dos últimas décadas, España ha pasado de ser un país emisor de emigrantes a ser un país receptor de inmigrantes de otros países y continentes, con los conflictos de integración que ello acarrea.

En este contexto, la Comunidad de Madrid, y más concretamente Madrid capital y su área metropolitana, aparecen como el destino más importante de los inmigrantes extranjeros en España, y muy especialmente de los procedentes de América Latina. Esta constatación justifica por sí misma la importancia del fenómeno.

Todo esto viene a reforzar la idea de la necesidad de una mejor integración de los inmigrantes en la sociedad española, para lo cual se hace imprescindible centrar una mayor atención en la evolución de estos nuevos colectivos y obtener la mayor información posible al respecto.

\subsection{Objeto de estudio, objetivos y justificación}

La presente investigación se ha centrado en la identificación y análisis de las demandas informativas de los inmigrantes latinoamericanos en la Comunidad de Madrid, como paso previo para un estudio más completo sobre los mecanismos del cambio social y cultural, pero desde una perspectiva comunicacional, es decir, partiendo del análisis del fenómeno de la mediación que interviene en los procesos de enculturización o socialización. Esta primera investigación parcial, pues, deberá servir para ulteriores estudios 
que se realicen, con mayor profundidad, sobre los usos de la comunicación y los procesos de mediación entre estos colectivos.

Por lo tanto, el objetivo general principal de la presente investigación se centró, fundamentalmente, en la identificación de los tipos de realidades sociales o aconteceres que son de interés para el colectivo de inmigrantes latinoamericanos. Los objetivos específicos de la investigación que hicieron posible la operación de identificar el acontecer público del endogrupo, es decir, los principales referentes un objetos de referencia sobre los cuales se informan los sujetos, reflejándose así las demandas informativas de los mismos, fueron los siguientes:

a) Descripción de las expectativas, aspiraciones, fines o metas de los sujetos

b) Descripción de los logros y frustraciones de los sujetos

c) Descripción de las necesidades y deseos de los sujetos

d) Descripción de los problemas y preocupaciones de los sujetos

e) Descripción de los intereses, aficiones, espacios y tiempos de ocio de los sujetos

Para este estudio se ha escogido el colectivo de inmigrantes latinoamericanos, por ser éste el más numeroso en España y en la Comunidad de Madrid, y conformar un grupo en gran medida homogéneo por sus características culturales y lingüísticas. Dentro de este grupo, el análisis se ha centrado en los inmigrantes procedentes de Colombia, Ecuador, Perú, República Dominicana, Bolivia y Argentina, ya que son los que mayor presencia tienen en la comunidad ámbito geográfico del estudio (Ver Tabla I). Ello no es óbice para que, en ulteriores investigaciones, puedan abordarse estudios similares sobre colectivos de otros países.

Por otra parte, el estudio se limitó al ámbito geográfico de la Comunidad de Madrid por razones obvias, ya que es esta comunidad autónoma, junto con la de Cataluña, la que concentra el mayor número de extranjeros residentes en España.

La elección como objeto de estudio de las demandas informativas como investigación previa a la de los usos de la comunicación, se debió al hecho de tratarse de una línea de investigación escasamente abordada dentro del área de las Ciencias de la Comunicación.

Aunque no es la primera vez que se realizan estudios sobre demandas y usos de la comunicación (Martín Serrano, 1983), la presente investigación, aun partiendo de los mismos presupuestos, es original y en cierto modo pionera, en cuanto al tratamiento de la integración del colectivo de inmigrantes en España desde una perspectiva comunicacional de los procesos de enculturización. Así mismo tampoco abundan las investigaciones que utilizan técnicas cualitativas para analizar el colectivo inmigrante (Martínez, Pascual, 2006), (Labrador, 2001) (González, Álvarez-Miranda, 2005) y apenas es utilizada la técnica del grupo de discusión (Sastre, 2005). Este trabajo utilizó, entre otras técnicas, el grupo de discusión con 
inmigrantes en la Comunidad de Valencia, llegando a conclusiones para el caso de los inmigrantes latinoamericanos congruentes con las expuestas en este estudio.

Asimismo, creemos que este estudio es de una gran utilidad práctica, por cuanto el abordaje de la integración desde una perspectiva enculturizadora, ayuda a entender mejor estos procesos con vistas a la creación de programas y proyectos institucionales y/o privados que puedan servir para la intervención en las acciones integradoras de los extranjeros residentes en España. En este sentido, el estudio cumple una función pragmática en el contexto de la investigación para el desarrollo ( + D), siendo de gran interés estratégico, tanto para instituciones públicas como privadas, en la medida en que informan a éstas sobre diversos aspectos del colectivo objeto de estudio.

Desde un punto de vista estrictamente científico, la investigación aporta también un importante avance en el conocimiento científico dentro del área de estudio de las Ciencias de la Comunicación. El estudio es perfectamente viable a nivel teórico, metodológico y práctico.

\subsection{Metodología de la investigación}

Para el abordaje de este estudio hemos utilizado una investigación aplicada de tipo empírico, utilizando una perspectiva cualitativa. Considerando ese carácter empírico de la investigación, se utilizaron básicamente fuentes primarias, basadas en la observación directa de los fenómenos, aunque como en toda investigación, sea ésta empírica o no, hemos llevado a cabo una primera fase documental sirviéndonos de fuentes secundarias, exclusivamente en los capítulos referentes al fenómeno de la inmigración en España y a la cuestión del objeto de referencia como parte del subsistema de Comunicación vinculado al Sistema Social. Esto explica la ausencia de citas en el capítulo dedicado a los resultados del estudio empírico, pues éste era el objetivo fundamental de este artículo, es decir, mostrar los fenómenos que habían surgido al abordar el estudio de campo y para lo que, es obvio, no existen fuentes no siendo las primarias.

También hemos llevado a cabo un tipo de diseño exploratorio de acuerdo con las características de nuestro objeto de estudio. En palabras de Santesmases, entendemos por estudio exploratorio "un estudio preliminar, muy flexible y poco formal, que se basa en el estudio de datos ya existentes, entrevistas con personas expertas y en el examen de situaciones análogas, mediante casos de estudio y simulaciones" (2004: 60).

En nuestro caso, la finalidad principal del diseño exploratorio fue descubrir ideas que pudiesen identificar problemas y/o variables relevantes para ser aplicados en una segunda fase investigadora sobre los usos de la información por los inmigrantes. Gracias a este diseño exploratorio hemos conseguido delimitar perfectamente aquellas demandas informativas que necesitábamos saber para, en una posterior investigación, analizar qué fuentes de información y qué usos de la información harían los inmigrantes latinoamericanos sobre cada una de esos objetos de referencia identificados. 
El diseño no podía ser ni descriptivo, ni causal, por la razón de que en esta primera investigación no era nuestro objetivo verificar hipótesis previas sino que pretendíamos, a partir de un diseño exploratorio, descubrir posibles hipótesis futuras. Esta es también la razón de que no hayamos considerado oportuno utilizar un método de abordaje hipotético-deductivo, que sí será utilizado en una fase posterior de este proyecto, cuando analicemos los usos de la información de los inmigrantes latinoamericanos partiendo del conocimiento de sus demandas informativas.

Para el presente estudio se utilizó, como procedimiento, el método cualitativo, que podríamos definir, apelando a las palabras de Miguel Beltrán, como "el propio discurso que se constituye en el objeto privilegiado de la investigación; el lenguaje no sólo como instrumento para investigar la sociedad, sino el objeto propio del estudio" (Citado por: García, 1994: 41). Definición que como vemos, difiere radicalmente del concepto metodológico cuantitativista.

Partiendo de un método cualitativo, la técnica de investigación utilizada fue la dinámica de grupos, más concretamente el grupo de discusión, siguiendo el modelo utilizado por Jesús Ibáñez en Más allá de la sociología. El grupo de discusión: Técnica y crítica (1992). El funcionamiento del mismo ha tenido en cuenta los dos elementos clave de esta técnica de investigación: los personajes o miembros del grupo, y el escenario.

Se realizaron tres grupos de discusión, compuestos cada uno por diez sujetos. Se contactó con estos por intermediación de diversas asociaciones de inmigrantes, y se seleccionaron a los individuos que respondieran al perfil. Un primer grupo lo constituyeron únicamente inmigrantes latinoamericanos del género masculino; un segundo grupo estuvo formado por inmigrantes latinoamericanos del género femenino, y un tercero estuvo compuesto por inmigrantes de ambos géneros. Seguimos estos criterios para homogeneizar los grupos. De esta forma podríamos identificar los temas de interés de los hombres y de las mujeres por separado, de manera que en la discusión la presencia de uno y otro género no influyese en los discursos. El tercer grupo constituido, en el que se mezclaron a hombres y mujeres, se realizó como una tercera alternativa experimental con la finalidad de ver si los discursos coincidían con la presencia de ambos géneros enfrentados.

Para que los miembros del grupo fueran lo más representativos posible, los protagonistas de los grupos no fueron seleccionados aleatoriamente, sino atendiendo al peso demográfico de las diferentes nacionalidades de los inmigrantes residentes en la Comunidad de Madrid. De esta forma, siempre hubo cuatro ecuatorianos-as, dos colombianos-as, un peruano-a, un boliviano-a, un dominicano-a, y un argentino-a. (Ver Tabla I).

Como hemos visto, el único criterio sociológico a la hora de constituir los grupos ha sido el género de los integrantes por no considerar necesario otro tipo de criterio. Casi el $60 \%$ de los inmigrantes latinoamericanos en Madrid se encuentran entre los 20 y los 40 años (ver Tabla II), constituyendo por 
ello un grupo muy homogéneo atendiendo a esa variable. Hay que tener en cuenta que en los países latinoamericanos subdesarrollados y en vías de desarrollo la mayor parte de la población es joven, y quienes emigran lo son en mayor grado. Crear grupos alternativos atendiendo a la edad hubiera exigido multiplicar los grupos de discusión, pues el haber introducido diferentes grupos de edad en un mismo grupo hubiese sido contraproducente, ya que los grupos de discusión deben ser homogéneos en su composición. Tampoco se consideró crear grupos de discusión atendiendo a la clase social o situación económica, por las mismas razones anteriormente citadas, es decir, la mayor parte de los inmigrantes latinoamericanos en España pertenecen a las clases sociales más desfavorecidas, siendo muy minoritarios los casos de estudiantes y de profesionales altamente cualificados. En relación con el tiempo de estancia de los inmigrantes en España, no hemos considerado pertinente hacer grupos de discusión en función de los que llevaban poco tiempo o mucho tiempo en el país, a tenor del objetivo de nuestra investigación. Por ello los miembros del grupo seleccionados fueron sujetos que llevaban en España más de un año, lo que representa el 94\% de la población inmigrante latinoamericana en Madrid (Ver Tabla III).

Atendiendo al escenario de las discusiones de grupos, hay que considerar tres elementos: el investigador o investigadores que participan en la dinámica del grupo, las relaciones entre los miembros y el lugar o espacio de las reuniones.

Los tres grupos de discusión se llevaron a cabo con la presencia de tres investigadores que actuaron del mismo modo en los tres casos. Dos de los investigadores compartieron mesa con los miembros de los grupos, separados ambos por un espacio prudencial y mezclados con los componentes del grupo. Un tercer investigador se situó alejado en un espacio exterior al del grupo con el único objetivo de observar los aspectos no lingüísticos de la discusión (captación de gestos, disposiciones, situaciones implícitas generadas, etc.).

Uno de los investigadores se encargó de exponer a los miembros de los grupos los objetivos de la investigación, mientras que el otro investigador fue el encargado de llevar a cabo la "provocación inicial", es decir, la propuesta o propuestas a discutir. Las propuestas de las discusiones fueron connotadas (no denotadas), es decir, se propusieron unos temas a un nivel superior, preguntando indirectamente por cuestiones de índole psicológica para alcanzar la finalidad de conocer el objeto de nuestra investigación: los temas de interés de los inmigrantes latinoamericanos.

Los temas o provocaciones que se utilizaron, en diversos momentos de la reunión, estaban explicitados en una lista-guía que se había elaborado previamente. Esas cuestiones que deberían generar la provocación para el arranque de la discusión fueron cuatro, y se corresponden con los objetivos específicos de la investigación:

a) Metas y expectativas en España

b) Necesidades y deseos 


\section{c) Problemas y preocupaciones \\ d) Hobbies}

Como es común en los grupos discusión la participación de los investigadores en la dinámica de los grupos fue mínima, dejando hablar a estos libremente a partir del silencio por parte del investigador una vez provocado el tema a ser debatido. Los investigadores sólo intervenían en momentos puntuales, cuando los miembros del grupo se desviaban del asunto, y con la única finalidad de reorientar la cuestión a ser debatida. A lo largo de las discusiones de grupo se generaron los problemas típicos en este tipo de dinámicas, como fueron las digresiones por parte de los miembros, el monopolio de la palabra de forma exagerada por alguno de los miembros y la escasa participación verbal de algún miembro.

La discusión de grupos se realizó en el interior de las instalaciones del Instituto de la Democracia, adscrito a la Universidad San Pablo-CEU, habiendo sido grabados los discursos de los miembros de los grupos. El hecho de que el escenario fuese un instituto de investigación adscrito a una universidad daba prestigio al acto y al mismo tiempo confianza a los miembros de los grupos. Las sesiones se llevaron a cabo alrededor de una mesa ovalada, lo que según los teóricos de los grupos de discusión como el ya citado Jesús Ibáñez, dan circularidad y unión en las relaciones entre los miembros, lo que siempre es positivo para la buena marcha de la discusión.

Como es común en este tipo de técnica, las discusiones de los grupos fueron grabadas en audio y video, ocupando el micrófono el centro de la mesa y situada la cámara en el extremo de la sala a una considerable distancia. Posteriormente, los discursos fueron transcritos, interpretados y analizados, siguiendo el padrón oficial para este tipo de técnica de investigación, como indica Jesús Ibáñez:

En el grupo terapéutico, la interpretación está supeditada al análisis. En el grupo de discusión el análisis está supeditado a la interpretación. El preceptor analiza el discurso del grupo y a partir de su análisis construye interpretaciones mediante las que capturar a los clientes (consumidores o votantes (Ibáñez, 1992: 577)

La interpretación del discurso se llevó a cabo siguiendo criterios semiológicos, es decir, se identificó la intención del discurso, lo que se quiere decir y está implícito en el discurso, teniendo en cuenta también los contextos lingüístico y extralingüístico. Dentro de este último contexto se tuvo en cuenta muy especialmente una interpretación de la situación o frame, subrayando la posición de los miembros del grupo, los valores que esgrimían, sus gestos, sus silencios, etc.

Una vez interpretado el discurso se pasó a su análisis definitivo, siguiendo una técnica de análisis crítico-impresionista, deduciendo los temas de interés u objetos de referencia de los grupos a partir de sus discursos acerca de sus fines y metas, necesidades y deseos, problemas y preocupaciones y hobbies, como quedó reflejado en el último capítulo de este artículo. 


\section{La inmigración latinoamericana en la Comunidad de Madrid}

Según la revisión del Padrón Municipal de 2005 para los municipios de la Comunidad de Madrid, a 1 de enero de 2005 el número de inmigrantes censados procedentes de Latinoamérica era de 413.778 (189.984 varones y 223.794 mujeres). La distribución por nacionalidades en datos porcentuales era como sigue:

Tabla I

Inmigrantes latinoamericanos por sexo y nacionalidades en la Comunidad de Madrid

\begin{tabular}{|c|c|c|c|c|c|c|c|c|}
\hline & \multicolumn{2}{|c|}{ Ambos sexos } & \multicolumn{2}{|c|}{ Varones } & \multicolumn{2}{|c|}{ Mujeres } & \multirow{2}{*}{\begin{tabular}{|c|}
$\%$ \\
Hombres \\
\end{tabular}} & \multirow{2}{*}{$\begin{array}{c}\% \\
\text { Mujeres }\end{array}$} \\
\hline & $\%$ & Absolutos & $\%$ & Absolutos & $\%$ & Absolutos & & \\
\hline Costa Rica & 0,08 & 346 & 0,09 & 169 & 0,08 & 177 & 48,84 & \\
\hline Cuba & 2,09 & 8.660 & 2,28 & 4.339 & 1,93 & 4.321 & 50,10 & 49,90 \\
\hline El Salvador & 0,20 & 808 & 0,17 & 315 & 0,22 & 493 & 38,99 & 61,01 \\
\hline Guatemala & 0,13 & 556 & 0,11 & 211 & 0,15 & 345 & 37,95 & 62,05 \\
\hline Honduras & 0,25 & 1.050 & 0,19 & 364 & 0,31 & 686 & 34,67 & 65,33 \\
\hline Nicaragua & 0,13 & 518 & 0,10 & 187 & 0,15 & 331 & 36,10 & 63,90 \\
\hline Panamá & 0,10 & 420 & 0,11 & 205 & 0,10 & 215 & 48,81 & 51,19 \\
\hline Rep. Dominicana & 5,75 & 23.785 & 5,28 & 10.034 & 6,14 & 13.751 & 42,19 & 57,81 \\
\hline Resto América Central & 0,08 & 311 & 0,06 & 120 & 0,09 & 191 & 38,59 & 61,41 \\
\hline México & 1,62 & 6.710 & 1,55 & 2.941 & 1,68 & 3.769 & 43,83 & 56,17 \\
\hline Argentina & 5,16 & 21.367 & 5,64 & 10.721 & 4,76 & 10.646 & 50,18 & 49,82 \\
\hline Bolivia & 6,43 & 26.589 & 6,00 & 11.406 & 6,78 & 15.183 & 42,90 & 57,10 \\
\hline Brasil & 2,46 & 10.172 & 2,41 & 4.583 & 2,50 & 5.589 & 45,06 & 54,94 \\
\hline Colombia & 17,55 & 72.636 & 17,23 & 32.725 & 17,83 & 39.911 & 45,05 & 54,95 \\
\hline Chile & 2,04 & 8.430 & 2,24 & 4.247 & 1,87 & 4.183 & 50,38 & 49,62 \\
\hline Ecuador & 41,95 & 173.593 & 42,68 & 81.093 & 41,33 & 92.500 & 46,71 & 53,29 \\
\hline Paraguay & 1,26 & 5.227 & 0,91 & 1.735 & 1,56 & 3.492 & 33,19 & 66,81 \\
\hline Perú & 9,49 & 39.274 & 9,67 & 18.378 & 9,34 & 20.896 & 46,79 & 53,21 \\
\hline Uruguay & 0,83 & 3.449 & 0,94 & 1.784 & 0,74 & 1.665 & 51,73 & 48,27 \\
\hline Venezuela & 2,39 & 9.873 & 2,33 & 4.425 & 2,43 & 5.448 & 44,82 & 55,18 \\
\hline Resto América del Sur & 0,00 & 4 & 0,00 & 2 & 0,00 & 2 & 50,00 & 50,00 \\
\hline Totales & 100 & 413.778 & 100 & 189.984 & 100 & 223.794 & 45,91 & 54,09 \\
\hline
\end{tabular}

Fuente: Revisión del Padrón Municipal de 2005. Instituto Nacional de Estadística. 
Por tanto la inmigración de Ecuador, Colombia, Perú, Bolivia, República Dominicana y Argentina, supone el $86,3 \%$ del total de inmigrantes latinoamericanos. Esta ha sido la nacionalidad de los individuos seleccionados para la realización de los grupos de discusión, procurando que su presencia muestral sea lo más aproximada posible a su representación en la población.

Si consideramos la población de estos países comprobamos que las naciones que tiene un mayor porcentaje de emigración en Madrid son por orden Ecuador (el 1,33\% de su población), Bolivia (un 0,3\%), República Dominicana $(0,27 \%)$, Colombia $(0,16 \%)$ y Argentina $(0,06 \%)$. Este orden se corresponde con el atractivo de Madrid como destino de emigración para la población de esos países. Se percibe el efecto llamada que hace que los inmigrantes ecuatorianos sean un porcentaje reseñable de la población ecuatoriana.

Respecto a la presencia de hombres y mujeres, hay mayor presencia de éstas con una diferencia de un $8 \%$. De las nacionalidades de presencia mayoritaria aquella en la que hay una mayor diferencia es la dominicana, con casi un $15,6 \%$; le siguen la boliviana (14,2\% a favor de las mujeres), la colombiana (casi un $10 \%)$, Ecuador $(6,6 \%)$, Perú $(6,4)$ y la menor la argentina, con una ligera predominancia masculina. El fenómeno de la mujer emigrante es un hecho que habrá que considerar.

Respecto a la distribución por edades en las nacionalidades mayoritarias es como sigue:

Tabla II

Inmigrantes latinoamericanos por edades y nacionalidades mayoritarias en la Comunidad de Madrid

\begin{tabular}{|l|r|r|r|r|r|r|r|r|r|r|r|}
\cline { 2 - 11 } & Total & $\begin{array}{c}\text { Hasta } \\
\mathbf{1 4} \text { años }\end{array}$ & $\mathbf{1 5 - 1 9}$ & $\mathbf{2 0 - 2 4}$ & $\mathbf{2 5 - 2 9}$ & $\mathbf{3 0 - 3 4}$ & $\mathbf{3 5 - 3 9}$ & $\mathbf{4 0 - 4 4}$ & $\mathbf{4 5 - 4 9}$ & $\mathbf{5 0 - 5 4}$ & $>\mathbf{5 4}$ \\
\hline Rep. Dominicana & 23.785 & 3.890 & 2.622 & 2.688 & 3.038 & 3.259 & 2.778 & 1.941 & 1.314 & 805 & 1.450 \\
\hline Argentina & 21.367 & 2.923 & 818 & 1.446 & 3.763 & 3.665 & 2.244 & 1.707 & 1.282 & 988 & 2.531 \\
\hline Bolivia & 26.589 & 3.187 & 1.079 & 4.278 & 5.702 & 4.585 & 3.032 & 2.194 & 1.306 & 660 & 566 \\
\hline Colombia & 72.636 & 10.398 & 3.868 & 6.924 & 11.062 & 11.134 & 10.237 & 7.841 & 4.889 & 2.862 & 3.421 \\
\hline Ecuador & 173.593 & 33.661 & 10.334 & 21.664 & 30.048 & 26.542 & 19.638 & 13.675 & 8.308 & 4.605 & 5.118 \\
\hline Perú & 39.274 & 3.235 & 1.914 & 3.866 & 6.156 & 6.503 & 5.624 & 4.175 & 2.868 & 1.721 & 3.212 \\
\hline TOTAL Latino-amer. & 413.778 & 63.389 & 22.998 & 46.809 & 70.118 & 65.449 & 50.503 & 36.704 & 23.227 & 13.859 & 20.722 \\
\hline \% sobre total & & $15,3 \%$ & $5,6 \%$ & $11,3 \%$ & $16,9 \%$ & $15,8 \%$ & $12,2 \%$ & $8,9 \%$ & $5,6 \%$ & $3,3 \%$ & $5,0 \%$ \\
\hline TOTAL Población & 413.778 & 63.389 & 22.998 & 46.809 & 70.118 & 65.449 & 50.503 & 36.704 & 23.227 & 13.859 & 20.722 \\
\hline \% sobre total & & $10,25 \%$ & $3,68 \%$ & $5,03 \%$ & $6,83 \%$ & $7,00 \%$ & $6,42 \%$ & $5,77 \%$ & $5,00 \%$ & $4,28 \%$ & $17,75 \%$ \\
\hline
\end{tabular}

Fuente: Revisión del Padrón Municipal de 2005. Instituto Nacional de Estadística. 
Respecto a la distribución por edades de la población española destaca la mayor presencia entre los inmigrantes de adultos jóvenes y niños. El grupo quinquenal más abundante en comparación con el total de población se encuentra entre los 30 y 34 años, le sigue entre 25 y 29, 20 y 24 y a continuación 35 a 39, 40 a 44 . El bloque de edad mayor de 50 años es menor, diferencia más acentuada según aumenta la edad.

La mayor presencia de niños obedece no sólo a la mayor tasa de fecundidad de la población inmigrante sino a los procesos de reunificación familiar con los hijos menores de edad. La población inmigrante se distribuye según una curva normal en torno a los 30 a 34 años. La menor presencia de mayores de 50 años indica por un lado que con la edad disminuyen las expectativas de progreso del emigrante, por un lado, y que la reunificación no se produce con los ascendientes, que rara vez abandonan su patria de nacimiento.

Por último se consideró relevante para elaborar los grupos de discusión conocer el tiempo de permanencia de los latinoamericanos en España, ya que esto afecta de manera acusada a la percepción de sus necesidades.

Tabla III

Extranjeros residentes en España provenientes de América Latina por tiempo de permanencia en España

\begin{tabular}{|l|c|c|}
\hline Menos de 1 año & 95,2 & $6,0 \%$ \\
\hline 1 año & 157,9 & $9,9 \%$ \\
\hline 2 años & 246,5 & $15,5 \%$ \\
\hline 3 años & 279,9 & $17,6 \%$ \\
\hline De 4 a 6 años & 657,3 & $41,3 \%$ \\
\hline 7 años o más & 156 & $9,8 \%$ \\
\hline
\end{tabular}

Fuente: Encuesta de Población Activa IV Trimestre de 2005. Instituto Nacional de Estadística.

Elaboración propia.

Los datos para el total de España determinan que el grueso de los inmigrantes latinoamericanos lleva en España entre 4 y 6 años, tiempo suficiente para haber culminado el proceso de regularización legal. Se percibe un pico de emigración latinoamericana tres años atrás desde el último cuatrimestre de 2005, situándonos en el 2002. Esto puede responder a la percepción de un momento álgido de la crisis económica latinoamericana y al efecto llamada de la regularización del 2000 y 2002. 


\section{El objeto de referencia en el proceso comunicacional}

El estudio de la sociedad humana, entendida tradicionalmente como un sistema adaptativo de enorme complejidad, viene siendo abordado por las ciencias sociales a través del análisis de tres subsistemas que le son inherentes: el sistema social propiamente dicho, como un ordenamiento de las relaciones que se establecen entre los miembros del grupo; el sistema cultural, conceptuado como el conjunto de reglas y normativas que orientan las relaciones, posicionamiento y funciones de los miembros del grupo dentro del sistema; y el sistema de conducta individual, vinculado a las características biológicas, psicológicas y a la experiencia de los individuos que conforman ese sistema. Partiendo de esta clasificación, la sociedad humana puede ser analizada, por tanto, desde perspectivas sociales, culturales y psicológicas.

El sistema de relaciones sociales que configura la estructura organizativa de las sociedades, se manifiesta a través de un conjunto de instituciones, constituyendo subsistemas o estructuras interconectadas en función de normas y reglas institucionalizadas que subyacen en el sistema cultural. El número de miembros, grupos e instituciones sociales; la posición que ocupan dentro del sistema, y las funciones y roles que desempeñan dentro del sistema, determinan el tipo de organización social de que se trate. En este sentido, hay que subrayar relaciones de parentesco, de poder y de dominación, de producción, de comunicación cultural, etc.

Por otra parte, el sistema cultural, que se articula con la organización social a través de los códigos o patrones que ordenan las relaciones entre los miembros del grupo, hay que entenderlo como el conjunto complejo de productos culturales simbólicos, en el sentido dado por Durkheim, es decir, el conjunto de representaciones cognitivas culturales y colectivas (representaciones sociales), constituidas por normas, creencias, mitos, sistemas de valores, etc.

Al mismo tiempo, habrá que considerar que el sistema cultural compartido dependerá, en última instancia, de las características psicológicas individuales de cada uno de los miembros del cuerpo social, aisladamente. Citando a Durkheim:

"Las representaciones colectivas son el producto de una inmensa cooperación, que no sólo abarca el tiempo, sino también el espacio; una multitud de espíritus diferentes ha asociado, mezclado, combinado sus ideas y sentimientos para elaborarlas (...) En el hombre hay dos seres: un ser individual, que tiene sus raíces en el organismo (...) y un ser social, que en nosotros representa la más elevada realidad, sea en el orden intelectual como en el orden moral, y que conocemos mediante la observación: me refiero a la sociedad" (1982: 14)

Marcel Mauss completa esta visión, cuando afirma que "la conciencia es un flujo continuo de las representaciones que se pierden, las unas con las otras, y cuando aparecen las distinciones, son completamente fragmentarias" (1971:16).

La sociedad humana, como todo sistema altamente complejo, es dinámico y, por tanto, sujeto a cambios en el tiempo. En todo proceso de transformación social es necesario distinguir modificaciones, tanto en la organización social, como en las innovaciones tecnológicas y los cambios culturales. 
Consideramos que en la estabilización de cualquier tipo de sociedad humana y su posterior evolución a través de los cambios en su composición y organización, intervienen múltiples factores, tanto externos -vinculados al ecosistema-, como internos, relacionados con aspectos demográficos, económicos, tecnológicos, culturales y psicográficos. Siguiendo a George M. Forster: "Podemos concebir la sociedad como sometida a dos tipos de cambios: los que tratan de promover los cambios y los que procuran conservar el status quo" (1988:94).

La continuidad del sistema, es decir, su reproducción, va a depender en gran medida de la adaptación de los elementos del sistema -colectividad de individuos- a las normas y reglas en forma de constricciones impuestas dentro del propio sistema. Para que eso ocurra, es necesario que exista una mínima coherencia entre organización, tecnología y cultura. El mecanismo que hace posible eso es el de los procesos de socialización (enculturización) y de aculturación (transculturación).

Partiendo de un proceso de enculturización. El sistema de valores predominante en una comunidad puede verse afectado por otro procedente del exterior. En palabras de Martín Serrano: "La producción social de comunicación es el punto de partida para estudiar las relaciones que existen entre transformación de la comunicación pública y el cambio de las sociedades" (1986:16). Este fenómeno puede ser estudiado en diversos tipos de sociedad, tanto en sociedades rurales agrarias que están transformando sus formas de vida tradicionales, como en colectivos de inmigrantes que se integran a una nueva sociedad. Sin embargo, desde siempre, en todo proceso de transformación social se observan contradicciones o disonancias, precisamente entre innovación tecnológica y cambio cultural.

Dentro del proceso comunicativo explicitado anteriormente, hay que destacar el Sistema Referencial (SR), íntimamente vinculado al Sistema Comunicacional (SC). El Sistema Referencial constituye el conjunto de temas o asuntos (objetos de referencia) sobre los que se elaboran los mensajes o contenidos de los discursos de los medios de comunicación de masas u otras fuentes de información. En otras palabras, el Sistema Referencial refleja el acontecer público que forma parte de la realidad o visión del mundo de los sujetos, y está constituido por elementos externos al propio sistema de comunicación:

"Los intercambios entre el subsistema de comunicación y el sistema social afectan a todos y cada uno de los componentes que intervienen en el proceso comunicacional. Esta interdependencia entre subsistema comunicacional y sistema social no puede ser ignorada a la hora de estudiar uno cualquiera de los componentes; $o$ dicho de otra forma, las investigaciones sobre emisores, receptores, medios de comunicación y sobre los mensajes que intercambian tienen que ser válidas por referencia a elementos exteriores al subsistema comunicacional, o no podrán ser nunca válidas" (Muñoz Carrión, 1978:57)

En este sentido, nuestro estudio no es un análisis de contenido de las informaciones o mensajes publicados que afectan a los inmigrantes latinoamericanos, ni siquiera un estudio sobre el uso que los inmigrantes hacen de las fuentes de información, sino que básicamente en esta investigación se abordan los referentes o asuntos sobre los cuales los sujetos se informan. 


\section{Las demandas informativas de los inmigrantes latinoamericanos}

En la construcción de las representaciones sociales o visión del mundo de una colectividad, que conforman el Sistema Cultural o Cognitivo (SCg.), además del Sistema Social (SS) y del Subsistema Comunicativo (SC) hay que contar con la influencia importante del denominado Sistema de Necesidades (SN) o motivacional de cada uno de los miembros que forman parte del etnogrupo y que representa los aspectos psicológicos que intervienen en el proceso comunicacional. El conjunto de todos estos aspectos psicológicos se traducen en una serie de intereses estratégicos hacia los que se supone que los miembros del etnogrupo se decantan, mostrando una especial atención.

Es en esta dimensión afectiva de los intereses colectivos en la que deben encuadrarse los referentes u objetos de referencia de la comunicación hacia los que los sujetos mostrarán sus inclinaciones a la hora de informarse. Estos referentes se traducen en demandas informativas, que a su vez conformarán el acontecer público del grupo objeto de estudio. Ahora bien, los objetos de referencia que conforman el Sistema Referencial (SR) en el proceso comunicativo, pueden imponerse ante la pasividad de los receptores, o pueden ser demandados por el público, transformándose en las citadas demandas informativas que constituyen la cosmovisión etnogrupal.

El Sistema de Necesidades (SN) a partir del cual hemos podido delimitar los objetos de referencia de los sujetos y, por tanto, sus demandas informativas, incluyó los siguientes elementos de análisis: los fines, metas o aspiraciones de los sujetos; sus necesidades y deseos; sus problemas y preocupaciones; y sus aficiones, lugares y tiempos de ocio.

\subsection{Las aspiraciones y metas de los inmigrantes}

De las discusiones de grupo se llegaron a una serie de resultados. Por un lado, las aspiraciones, metas, fines o expectativas del colectivo inmigrante latinoamericano, pueden clasificarse en tres categorías: aspiraciones referidas a prácticas o actividades; aspiraciones referidas a posesiones materiales, y aspiraciones de tipo existencial y social.

A lo largo de la investigación llevada a cabo se ha podido observar que son las aspiraciones de tipo existencial y social, en primer lugar, las de mayor prevalencia entre el colectivo de inmigrantes, muy especialmente fines o metas como la consecución del permiso de trabajo necesario para una legalización de estancia en el país, la adquisición de la nacionalidad española, el reagrupamiento familiar, la homologación de los estudios, la mejora del futuro de los hijos, una mejor integración en el país de acogida, rehacer la vida y prosperar.

Es importante destacar que la aspiración de prosperidad por parte de los inmigrantes está vinculada directamente con sus expectativas relacionadas con el viejo mito de España como país de oportunidades, existente en el imaginario del colectivo latinoamericano, es decir, la idea de España como protectora y 
donante de seguridad, trabajo y prosperidad, como así lo relataba uno de los miembros del grupo de discusión: "Yo llegaba acá a rehacer mi vida y estaba dispuesto a todo, en el sentido legal, digo. Entonces empecé a trabajar de coger y todo eso; pero siempre en Ecuador tuve la idea de que como éste [España] es un país de oportunidades, el conocimiento que teníamos, también uno tenía que poner parte en ese sentido.... yo me dije, bueno, voy a tratar de hacer lo que me gusta y borrar el típico estereotipo de sudamericano analfabeto, que trabaja en el campo y todo eso".

Obviamente, para los inmigrantes latinoamericanos, la prosperidad también implica una mejora en el futuro para sus hijos, la meta de que ellos sean "españoles" y puedan tener las oportunidades que sus padres no tuvieron, inclusive la consecución de la nacionalidad española como objetivo prioritario o, cuanto menos, la consecución del permiso de trabajo necesario para poder legalizar la estancia en el país, requisito sine qua non para obtener el tan anhelado empleo.

Para la mayor parte de los individuos tener estudios implica, en su imaginario, mejores oportunidades para ascender en la escala social, y también implica la posibilidad de conseguir mayores emolumentos, bienestar social y confort. Una parte de los inmigrantes objeto de estudio consideraba una salida la posibilidad de homologar los estudios con el objetivo de poder ejercer sus profesiones en España. Sin embargo no consideraron la opción de estudiar en España una vez asentados, pues "aquí se viene a trabajar".

La prosperidad en la comunidad de inmigrantes es asociado por los sujetos al éxito socioprofesional, la elevación en la posición social y la acumulación de capital. Cobra una especial relevancia montar un negocio, o dedicarse a un oficio y profesión cualificados.

Sin embargo, para la satisfacción de este fin -todavía percibido como una frustración o satisfacción no alcanzada- los inmigrantes latinoamericanos desgranan tres metas de naturaleza social indispensables, que no son otras que el propio reconocimiento de América Latina por España, por encima de las otras citadas a continuación; una mejor integración en el país de acogida, es decir, un mejor trato por parte de la población española; y el logro de una serie de derechos reivindicados por el colectivo de inmigrantes.

Por lo que respecta a las aspiraciones referidas a posesiones materiales, destacan fundamentalmente la posesión de dinero con la intención de ahorrar para invertir en los países de origen en el futuro. La acumulación de capital pecuniario como elemento de estabilidad y de inversión en el futuro es una de las metas mayormente expresadas. El dinero es percibido como un bien necesario, pero no se destina a un consumo ostentoso, sino al ahorro para asegurar el futuro y evitar calamidades. Esta conducta es más típica entre los inmigrantes más tradicionales procedentes del entorno rural de sus respectivos países. Para éstos, el dinero es una garantía existencial que les aleja de la miseria que experimentaron en el pasado, pues muchos de los inmigrantes mostraban temor a un posible retorno a la carestía de antaño. 
Los sujetos acumulan y depositan el dinero, generalmente, en los bancos y cajas de ahorro españolas en lugar de enviarlo a entidades financieras de sus países, por una cuestión de mayor confianza en las instituciones del país receptor. Los casos en que el inmigrante vive en el nivel de la subsistencia se producen, precisamente por el interés de gastar lo menos posible con la intención de poder regresar al país de origen.

Por otra parte, destaca el hecho de que la mayor parte de los inmigrantes no muestren una especial aspiración por consumir electrodomésticos, automóviles u otros productos de consumo más corrientes entre los españoles. Sin embargo, se hace perceptible la expectativa por adquirir una vivienda en España, sobre todo en el caso de aquellos inmigrantes que tienen unas expectativas de residencia en el país de más largo plazo o que tienen la intención de afincarse definitivamente en nuestro país.

En este sentido las aspiraciones referidas a prácticas o actividades tienen que ver con la mejora en las condiciones laborales y en una mayor estabilidad laboral en el futuro ante el fantasma del paro, así como la obtención de un mejor status social.

\section{2. $\quad$ Necesidades y deseos a partir de aspiraciones no satisfechas}

Toda aspiración, fin o meta de un sujeto implica una voluntad de satisfacer deseos y/o necesidades. Siguiendo la definición de Santesmases Mestre, entendemos por necesidad "una sensación de carencia de algo, un estado fisiológico o psicológico, que es común a todos los seres humanos, con independencia de los factores étnicos y culturales, siendo el deseo la forma en que se expresa la voluntad de satisfacer una necesidad" (2004: 50). Admitiendo esto último, hemos podido abordar las necesidades y deseos de los inmigrantes latinoamericanos a partir de fines y metas no satisfechos aún.

La presente investigación ha mostrado que el tipo de aspiraciones existenciales y sociales generan fundamentalmente necesidades y deseos de supervivencia entre los sujetos. De esta manera, la expectativa de conseguir un permiso de trabajo que legalice la situación en el país, genera la necesidad de una mayor agilidad burocrática y de una mayor facilidad en la consecución de la legalización de los inmigrantes. Los inmigrantes expresaron el deseo, entre otros, de que se pusiese fin a las colas en los consulados y una mayor facilidad también para la renovación del permiso de trabajo.

Por su parte la aspiración a conseguir la nacionalidad española demanda la necesidad de una mayor información sobre este asunto. Por ende, la expectativa de lograr una mejor integración en el país de acogida está ligada con la necesidad de que exista una mayor información en España de la realidad de los países latinoamericanos, lo que implica un mayor conocimiento por parte de los españoles de esa realidad y también una mayor información por parte de los inmigrantes de las noticias procedentes de sus países de origen.

Por lo que respecta a las aspiraciones referidas a posesiones materiales, que generan necesidades o deseos de tipo hedonista, destacan fundamentalmente la posesión de dinero con la intención de ahorrar 
para invertir en los países de origen en el futuro, la obtención de una vivienda digna y la obtención por parte de los sujetos de un mejor status social que el que tenían en sus países de origen.

Las aspiraciones referidas a prácticas o actividades tienen que ver con la mejora en las condiciones laborales y en una mayor estabilidad laboral en el futuro ante el fantasma del paro. Ello genera necesidades de proyección social que se relacionan directamente con el éxito, la prosperidad y el status social.

\subsection{Los intereses del etnogrupo a partir de los problemas y preocupaciones}

Hay que indicar que tanto las aspiraciones como las necesidades, satisfechas o frustradas, se interrelacionan directamente con los problemas y preocupaciones, que son a su vez indicadores importantes de los intereses del etnogrupo.

En este estudio se ha podido observar una clara identificación de preocupaciones que afectan directamente a los sujetos, y por lo tanto son de naturaleza claramente endogrupal, que fundamentalmente devienen problemas que se desean solucionar, aunque también se transforman en temas de interés. Sin embargo, prácticamente se ha observado una nula identificación de preocupaciones que no afectan directamente a los sujetos y que, por tanto, serían de de naturaleza exogrupal. En la visión del mundo construida por la colectividad, cabe diferenciar entre problemas, preocupaciones e intereses de naturaleza endogrupal, que surgen de la experiencia inmediata y cotidiana, de aquellos otros que son de naturaleza exogrupal, es decir, que provienen del contacto mediado con el mundo exterior.

Las preocupaciones e intereses endogrupales suelen estar más vinculados a los sujetos con un estilo de vida más tradicional; mientras que las preocupaciones e intereses exogrupales inciden más en sujetos con un estilo de vida no tradicional, es decir, relacionado más directamente con el concepto de modernidad. Los propios estilos de vida más tradicionales ejercidos por la mayor parte de los inmigrantes latinoamericanos en sus países de origen, explicarían en gran medida la hegemonía de problemas y preocupaciones de naturaleza endogrupal.

En la visión del mundo construida por la colectividad de inmigrantes, hemos podido diferenciar también entre problemas, preocupaciones e intereses de naturaleza existencial, y problemas, preocupaciones e intereses de naturaleza social relacionados con el problema de la integración en el país receptor. Dentro de los problemas y preocupaciones de naturaleza existencial hemos identificado los siguientes:

a) La situación de los inmigrantes en España como consecuencia de la actual legislación de Extranjería. Estas cuestiones están relacionadas con las siguientes cuestiones:

- La mayor o menor dificultad de acceder a un visado de permanencia en España, sea por la vía de la legalización laboral o por la vía de la consecución de un visado de permanencia como estudiantes. 
- La mala atención policial, acusándose a las comisarías españolas y a los propios consulados de los países originarios de los inmigrantes de una total desinformación. Esto crea una mala imagen burocrática española, percibiéndose entre los inmigrantes una falta de coordinación entre empresas, ayuntamientos, universidades, embajadas y consulados. Esto queda reflejado, por ejemplo, en las palabras de uno de los miembros de los grupos de discusión: "Empecé a dar vueltas para venirme, año y medio antes, y justo cuando ya tenía todo más o menos organizado, me dan cita en el consulado de Colombia... Entonces ya no sirve, esto es totalmente una situación ilógica, entonces habría que buscar otro tipo de sistemas y tratar de buscar voluntades en las embajadas".

- Pese a esto se percibe que es más fácil resolver las cuestiones burocráticas cuando se viene a trabajar que cuando se viene a estudiar. El extranjero que viene a estudiar se siente discriminado por las autoridades españolas y por su propia embajada y consulado, considerándose la situación humillante.

- La existencia de un posible tráfico de influencias para conseguir trabajo en España o poder acceder a realizar estudios en el país.

- La demora en los trámites burocráticos por parte de embajadas y consulados, sobre todo a la hora de tramitar los papeles de residencia o la propia nacionalidad española.

b) Cuestiones vinculadas con la situación laboral en España, como es el caso de preocupaciones relacionadas con la situación de subempleo, salarios bajos, explotación a que se ven sometidos los inmigrantes y el futuro laboral incierto como consecuencia de la posible competencia en el futuro inmediato de los países del Este de Europa. Estos problemas vienen expresados por una preocupación esencial por el futuro económico, laboral, profesional y existencial de los sujetos. También se advirtió que, aunque la mayoría de los inmigrantes latinoamericanos tienen como destino final España, se da el caso de algunas personas cuyo destino último es otro país, siendo España un país puente de entrada a países más desarrollados.

c) El acceso a la educación de los hijos y la homologación de los estudios también son preocupaciones que afectan directamente al colectivo latinoamericano. La primera preocupación se percibe como un problema en vías de solución a medio-largo plazo, manifestando una responsabilidad intrapunitiva, es decir, son conscientes de que la educación de los hijos va a depender de ellos directamente. Sin embargo, la homologación en España de estudios ya realizados en los países de origen se ve como un problema en vías de solución a más corto plazo, pero manifestándose una responsabilidad claramente extrapunitiva, ya que no depende directamente de ellos el logro de ese fin.

d) El problema de la vivienda preocupa muy especialmente a los inmigrantes latinoamericanos establecidos en la Comunidad Autónoma de Madrid, por razones obvias relacionadas con la 
situación de especulación inmobiliaria existente en España actualmente. Íntimamente relacionada con esta cuestión está la preocupación de este colectivo por la facilidad o dificultad de conseguir créditos bancarios, así como su preocupación por las condiciones de las hipotecas y la subida de los tipos de interés, ya que de la discusión de los grupos se percibió un interés cada vez mayor por la compra de vivienda en España.

Por otro lado del estudio se desprende que, en lo que se refiere a los problemas, preocupaciones e intereses de naturaleza social, las cuestiones relacionadas con la integración de los inmigrantes en la sociedad española son las más relevantes. Podemos resumir las principales preocupaciones, diciendo que en la mayor parte de lo expresado por los miembros de los grupos de discusión, figuraban temas relacionados con el supuesto creciente racismo y xenofobia por parte de la población española, percibido por los inmigrantes de una manera muy preocupante. Los prejuicios y estereotipos que la población española tiene contra los latinoamericanos en general, considerados por éstos como una imagen negativa y que no se corresponde con la realidad, también es una cuestión que afecta mucho al colectivo objeto de estudio. Entre otros aspectos que dificultan la integración en España estarían la propia imagen que los latinoamericanos tienen de los españoles, caracterizada por la discriminación, la falta de educación, la grosería y el temperamento agresivo de los españoles.

Por último es muy relevante señalar que una cuestión que afecta a la integración de los inmigrantes latinoamericanos en la sociedad española es la escasa y deficiente información que los medios de comunicación españoles dan sobre la situación política, socioeconómica y cultural de los países de origen de los inmigrantes. Esta preocupación tiene una doble dimensión. Por un lado existe una queja de esa falta de información que afecta a los propios inmigrantes. Esta es una de las causas que han favorecido en los últimos años la aparición de diversos medios de comunicación destinados a inmigrantes latinoamericanos y realizados por inmigrantes latinoamericanos, fundamentalmente medios de comunicación impresos y radiofónicos. Y por otra, la reivindicación de que los propios españoles deberían de informarse más sobre la realidad de sus países.

\subsection{Los hobbies, tiempos y espacios de ocio}

La mayor parte de los trabajadores latinoamericanos, fundamentalmente vinculados a los sectores de la construcción y de los servicios, son trabajadores asalariados, muchos de ellos pluriempleados, que apenas cuentan con tiempo libre para practicar sus aficiones. Mucho menos aún proliferan las prácticas ociosas que requieren gastos onerosos. El ahorro como meta esencial para conseguir un futuro mejor, limita la dimensión lúdica de naturaleza hedonista, y reafirma la dimensión laboriosa y productiva de naturaleza auto-represora.

Los tiempos de ocio son escasos, quedando relegados a horas determinadas del día y a determinados días de la semana, especialmente sábados y/o domingos. El ocio se centra fundamentalmente en prác- 
ticas de tipo pasivo, como son ver la televisión, un vídeo, oir la radio, oir música y asistir a fiestas privadas en viviendas particulares o en parques públicos, donde se concentran otros compatriotas para conversar y cambiar impresiones.

El ocio de tipo activo es menos practicado que el anterior, ya que implica un traslado a espacios de ocio determinados que no son el propio hogar y unos gastos que tratan de evitarse. Destacan especialmente la asistencia al cine, conciertos de música, bares y discotecas de ambiente latino y la práctica de deportes al aire libre. En este sentido, puede afirmarse que los principales intereses relacionados con el ocio son la música y el baile -de forma activa y pasiva, destacando músicas y ritmos de baile latinos-, el fútbol como deporte mayoritario, y la audiencia de programas televisivos de entretenimiento, especialmente reality-shows, talk-shows, late-shows y musicales.

\subsection{Las demandas informativas}

Como resultado final del análisis de todos los criterios aplicados en la investigación, hemos observado que el colectivo de inmigrantes latinoamericanos de la Comunidad de Madrid sometido a observación, ha mostrado una percepción del acontecer público tanto de naturaleza endógena como exógena. El primer tipo de percepción (de naturaleza endógena) se traduce en una serie de temas de interés u objetos de referencia que son problemas y preocupaciones existenciales que afectan directamente a los sujetos. Estos referentes o demandas informativas son seis fundamentalmente:

- La legislación española de extranjería

- El trabajo

- El paro

- La vivienda

- El dinero

- Los estudios

El segundo tipo de percepción del acontecer público (de naturaleza exógena), se traduce en temas de interés u objetos de referencia que son problemas y preocupaciones que no afectan directamente a los sujetos. Estos referentes o demandas informativas los hemos sintetizado en una fundamentalmente:

- La integración social

En esta demanda informativa se incluirían todas las cuestiones relacionadas con los prejuicios, estereotipos, racismo, xenofobia e imagen negativa de América Latina por parte de los españoles.

Por último, hemos considerado pertinente incluir como demandas informativas relacionadas con las prácticas de ocio, las siguientes:

- La música y el baile 
- El fútbol

- Los programas de entretenimiento de la televisión

Una vez hemos identificado ya las principales demandas informativas de los inmigrantes latinoamericanos en la Comunidad de Madrid, el siguiente paso que nos proponemos llevar a cabo será un análisis de los usos de la comunicación o fuentes de información utilizadas por este colectivo, con el objetivo de saber qué canales utilizan para informarse de cada uno de los objetos de referencia descritos. Para ello tenemos prevista una ulterior investigación que consistirá en la realización de una encuesta sobre los usos de la comunicación de los inmigrantes latinoamericanos en la Comunidad de Madrid, basándonos en las demandas informativas que hemos identificado en el presente estudio.

\section{Referencias bibliográficas}

- Díez Nicolás, J. (2005): Las dos caras de la inmigración. Madrid: Ministerio de Trabajo y Asuntos Sociales, Subdirección General de Información Administrativa y Publicaciones.

- Durkheim, É. (1982): Las formas elementales de la vida religiosa. Madrid: Editorial Akal.

- Forster, G. M. (1988) Las culturas tradicionales y los cambios técnicos. México: FCE.

- García Ferrando, M.; Ibáñez, J.; Alvira, F. (1994): El análisis de la realidad social: métodos y técnicas de investigación. Madrid: Alianza Universidad Texto.

- González Enríquez, C.; Álvarez-Miranda, B. (2005): Inmigrantes en el barrio: un estudio cualitativo de opinión pública. Madrid: Ministerio de Trabajo y Asuntos Sociales, Subdirección General de Información Administrativa y Publicaciones.

- Ibañez, J. (1992). Más allá de la Sociología. El grupo de discusión: Técnica y Crítica. Madrid: Siglo XXI.

- (1994). Cómo se realiza una investigación mediante grupos de discusión. En: Garcia Ferrando, M.; Ibañez, J.; Alvira, F. El análisis de la realidad social: métodos y técnicas de investigación. Madrid: Alianza Universidad Texto.

- Labrador Fernández, J. (2001): Identidad e inmigración: un estudio cualitativo con inmigrantes peruanos en Madrid. Madrid: Universidad Pontificia Comillas de Madrid.

- Martín Serrano, M. (1983): El uso de la comunicación social por los españoles. Madrid: CIS.

- (1986): La producción social de la comunicación. Madrid: Alianza Editorial.

- Martínez Ballesteros, C.; Pascual Lindes, P. (2006) Cómo nos vemos: percepción entre la población autóctona e inmigrante en la ciudad de Córdoba. Córdoba: Fundación para el Desarrollo de los Pueblos de Andalucía. 
- Mauss, M. (1971) Institución y culto. Vol. 2. Seix Barral, Barcelona.

- Muñoz Carrión, A.P.; Perez Garcia, R.J; Sánchez Carrión, J.J. (1978). Análisis del Referente. En: Revista Reis, n. ${ }^{\circ}$ 3, julio-septiembre. Madrid: Centro de Investigaciones Sociológicas (C.I.S)

- Santamarina, C. (2005): Consumo y ocio de los inmigrantes latinoamericanos en España: un acercamiento desde la perspectiva cualitativa. Madrid: Ministerio de Trabajo y Asuntos Sociales, Subdirección General de Información Administrativa y Publicaciones.

- Santesmases Mestre, M. (2004): Marketing: conceptos y estrategias. Madrid: Ed. Pirámide.

- Sastre García, V. J. et. al. (2005): Las necesidades sentidas de los inmigrantes en la Comunidad Valenciana: estudio sociológico cualitativo y cuantitativo. Valencia: Centro de Estudios para la Integración Social y Formación de Inmigrantes, Fundación de la Comunidad Valenciana.

- Thalhammer, E. (2001): Actitudes hacia los grupos minoritarios en la Unión Europea: un análisis especial de la encuesta Eurobarómetro 2000 en nombre del Observatorio Europeo del Racismo y la Xenofobia. Viena: Observatorio Europeo del Racismo y la Xenofobia.

- Tornos, A; Aparicio, R.; Fernández García, M. (2004): El capital humano de la inmigración. Madrid: Instituto de Migraciones y Servicios Sociales.

- Veredas Muñoz, S.; Carabaña Morales, J. (1998) Las asociaciones de inmigrantes marroquíes y peruanos en la Comunidad de Madrid. Madrid: Universidad Complutense, Facultad de Ciencias Políticas y Sociología, Departamento Sociología III.

- Villena Rodríguez, M. (2004): Demografía, mercado de trabajo y política de inmigración España vs U.E. Málaga: Eumed,

- Zamora, J. A.(2003): Ciudadanía, multiculturalidad e inmigración / Foro "Ignacio Ellacuria" Solidaridad y Cristianismo. Estella (Navarra): Verbo Divino. 
